\title{
CHARACTER RECOGNITION FOR BI-LINGUAL MIXED-TYPE CHARACTERS USING ARTIFICIAL NEURAL NETWORK
}

\author{
Rekha Singh ${ }^{1}$ \\ ${ }^{I}$ M.Tech Scholar, Department of Computer Science, Saroj Institute of Engineering \& Technology, Lucknow, India
}

\begin{abstract}
Artificial Intelligence has evolved to a great extent since its inception and is spreading wide day to day. Today, nearly all aspects of human life have a sound presence of some sort of application of artificial intelligence. A key role in this is of Artificial Neural network, a very vital branch of Artificial Intelligence. A vast majority of day by day applications have their evolvement from Artificial Neural Network, especially applications developed for those tasks which were earlier believed to be best performed by only human beings. This potential of ANNs lies in its degree of simplicity and generality; bringing the difference down in between, Human mind capability and machines.

This paper basically deals with recognition of isolated machine print as well as hand-written characters using artificial neural networks. The important aspect of this paper which makes it different from similar works in this field is that I have approached this problem with bilingual character set. I have approached to the recent trend where people are at home with use of English as well as Hindi language, not only in speaking but also in writing. Due to this, my focus has been to device network with almost equal capability of recognizing characters in both scripts; Devnagri and English. Besides this, text content faced by us in day to day is a mixture of type written and hand written. So the characters are either computer generated or by human handwriting. For this purpose data collection, feature extraction and neural network designs have been achieved. The training phase has successfully been completed with 790 samples of handwritten samples computer generated font styles. The overall performance of the system has been achieved more than 95\%, quite significant in case of human handwriting.
\end{abstract}

Keywords: Artificial Neural Network; Character, Recognition; Feed-forward Back-propagation Network $* * *$

\section{INTRODUCTION}

Machine simulation of human functions has been a very challenging research area since the advent of digital computers. In some areas which require certain amount of intelligence, such as number crunching or chess playing, tremendous improvements are achieved. On the other hand, humans still outperform even the most powerful computers in the relatively routine functions such as vision. Machine simulation of human reading is one of these areas, which was the subject of intensive research for the last three decades, yet it is still too far from the final frontier [1] [3].

Character recognition has always been a special problem. The problem increases when we operate it in the offline mode [9] where we need to read characters from some document thus by performing feature extraction, one of the complex processes. We see a lot of work has been done in this area in the past few years. The solutions being proposed mainly use Artificial Neural Networks (ANN) for solving the problem. And, this is well justified as, in case of Humans this is also done by the Neural Network [3] which quite accurately perform the Character recognition.

The early work on the automatic recognition of characters has been concentrated either upon well printed text or upon small set of well distinguished handwritten text or symbols. Successful, but, constrained algorithms had been implemented mostly for Latin characters and numerals.
Besides, some studies on Japanese, Chinese, Hebrew, Indian, Cyrillic, Greek and Arabic characters and numerals in both printed and handwritten cases have also been done[2][4].

Nowadays, there is a renewed interest in the character recognition field, which involves the recognition of both printed and handwritten characters [5]. It is not only that we now have more compact and powerful computers and more accurate electronic equipment such as scanner, camera, electronic tablet etc., but also we have better recognition algorithms which utilize advanced methodologies such as Neural Networks, Hidden Markov Models or Fuzzy Set Reasoning. But, still there is a long way to go in order to reach the ultimate goal of machine simulation of fluent human reading.

\section{THE PROPOSED APPROACH}

Handwriting recognition is a well-known problem which involves the recognition of whatever input is given in form of image, scanned paper, etc. The handwriting recognition generally involves the following steps, which are also been adapted in this work.

Scanning the document: A properly printed document is chosen for scanning A scanner software is invoked which scans the document. The document is sent to a program that saves it in preferably TIF, JPG or GIF format, so that the 
image of the document can be obtained when needed. This is the step in Optical Character Recognition. The size of the input image is taken as per the requirement of the feature extraction procedure.

Segmentation: This step deals with the breaking of the lines, words and finally getting all the characters separated. This step involves the identification of the boundaries of the character and separating them for further processing. In this approach adopted by me, first I have gone through line by line extraction of characters. After completing this line by line extraction, I have done with character by character extraction for each and every line applying the same approach as with line extraction but with a little change thus applying no extra emphasis for character extraction. This way, I have left with the inputs to the developed system as single characters.

Preprocessing \& Feature Extraction: This step involves the initial processing of the image, so that it can be used as an input for the recognition system. In this step, I had to concentrate over some issues which are quite important to make the input images unique as the input size of the neural network is always going to remain constant in terms of number of neurons in input layer. Therefore, each image should be of same size in terms of pixels, there dimensions should be same regardless the actual dimension of the character. For this reason, I have gone for a perfect square image.

Classification and Character Recognition: Once the input image is available in good condition, it may be processed for recognition. The role of the recognition system is to identify the character. The neural network designed in this work uses an image as an input for the same.

\section{IMPLEMENTATION}

Shown following is the procedure followed in the segmentation and pre-processing phase.

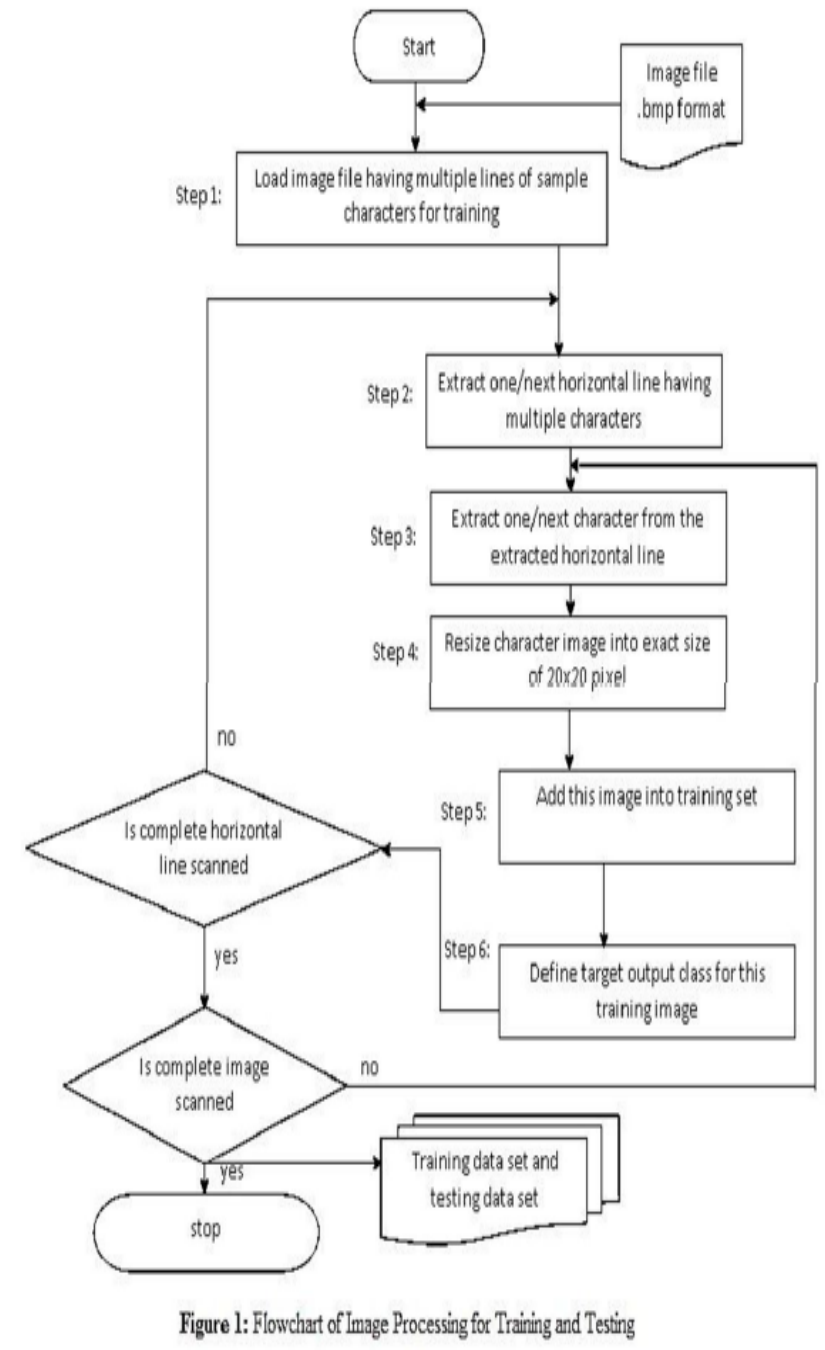

Step 1: For the network to train, the requirement has been set as an image file which may consists multiple lines and each line having multiple characters. The task prior to creation and training of a proper network is to generate a significant training set. Therefore, I have taken around 79 font styles for training of the network. In the initial stage, for the sake of measuring networks performance easily, the training set has limited to 10 characters only i.e. E, A, R, I, $\mathrm{O}$, d] j] g] l] u. And so, the number of training sample has gone to $79 \times 10=790$ samples.

Step 2: Once the image for training is ready, the next task is segmentation of the image in proper format. This is further broken in two phases. First, the segmentation is done in horizontal direction thus extracting line of characters. The basic rule for this is looking for line of white spaces and continuing till any horizontal line having signature of some character is found. Once, the line with signature of some character is found, we continue to scan horizontally till we keep founding signature of characters i.e. non-white space.

We stop when we found a horizontal line with only white spaces. It indicates that we have left with an image of having a line of characters. Now, the next step is to scan this new image to get the individual characters. We have also temporary stored each line of character. 
Step 3: Once an image of a single line having multiple characters in horizontal direction is achieved, the next phase is to apply same approach as of horizontal segmentation in vertical direction for each such line image and extract individual characters this time. For this, I have rotated the line image by 900 and applied logic of horizontal segmentation previously applied for achieving line image, thus getting vertical segmentation.

Step 4: After getting individual character images, the images are to be fed into neural network as input during the training phase and also in testing the network. The input size has to be remaining same during the whole process of training and also network has to be remaining same after completion of training. So, I have made each character same in size and dimension.

\section{EXPERIMENTAL RESULTS}

I have done my experimentation on feed forward back propagation network, which is till date the best proven network for application related with pattern recognition. In my character recognition work I have worked upon type written as well as hand written characters. More importantly, my work is based upon recognition of characters of not just one but two scripts simultaneously keeping the network unchanged.

In the following figures, I am showing the comparative ratio of success in recognition of English as well as Hindi characters which have been achieved during training phase. For this comparative study I have first, taken each character keeping same dimensions of $15 \times 15$ pixels. After which, I have kept dimension in 20x20 pixel; thus increasing range of input from 225 (15x15) to 400 (20x20). Following drawn are the results drawn. This is obvious that the feed forward back propagation network (FFBPN) is performing quite similar with very minor difference in their character-wise training performance for both the variation in image dimension (i.e. $15 \times 15$ and $20 \times 20$ ). This is also due to a sufficiently large training set. The above tables also show that FFBP Network in both of these cases has achieved training up to a saturation level in this phase. However, their actual performance is shown during the testing phase.

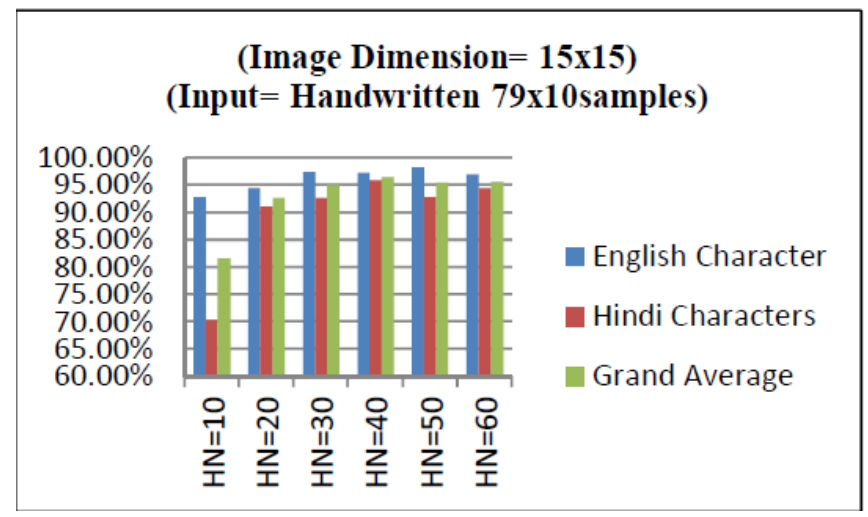

Fig 2: Comparative network performances for image Dimension $=15 \times 15$ for Handwritten 79x10samples

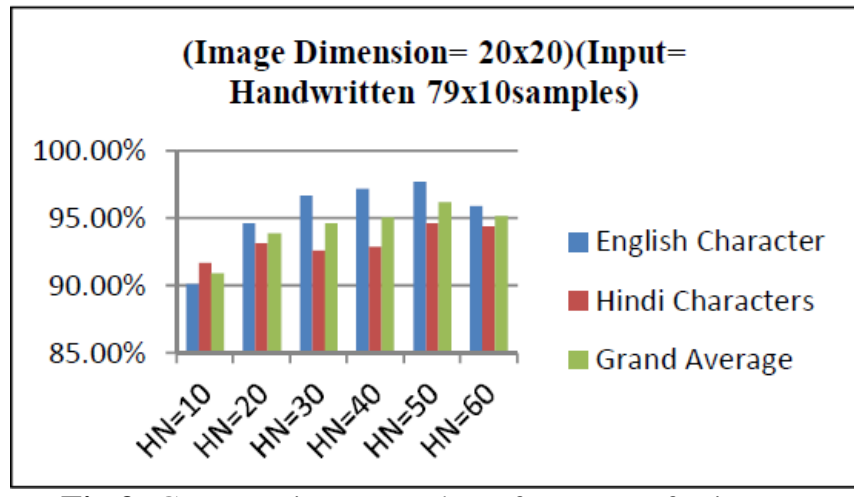

Fig 3: Comparative network performances for image Dimension $=20 \times 20$ for Handwritten 79x10samples

The network has achieved saturation on 40 Hidden layer neurons in case of image dimension of $15 \times 15$ while the saturation for dimension size $20 \times 20$ is achieved at 50 hidden layer neurons. This shows that as we increase the input size of the network, we get saturation on higher number of hidden layer neuron. However, we have also got very slight improvement in recognition rate on increasing input size.

After training performance on handwritten characters, I have performed the same operations with typewritten characters. For this a total number of 1000 (100 x 10) character samples have been taken. Below drawn in Fig are the results which consists result of this training.

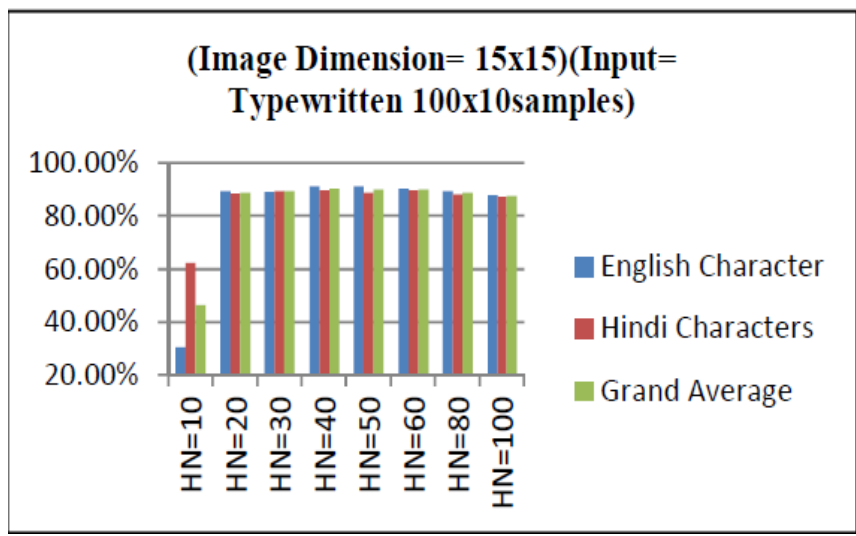

Fig. 4: Comparative network performances for image Dimension $=15 \times 15$ for Typewritten $79 \times 10$ samples

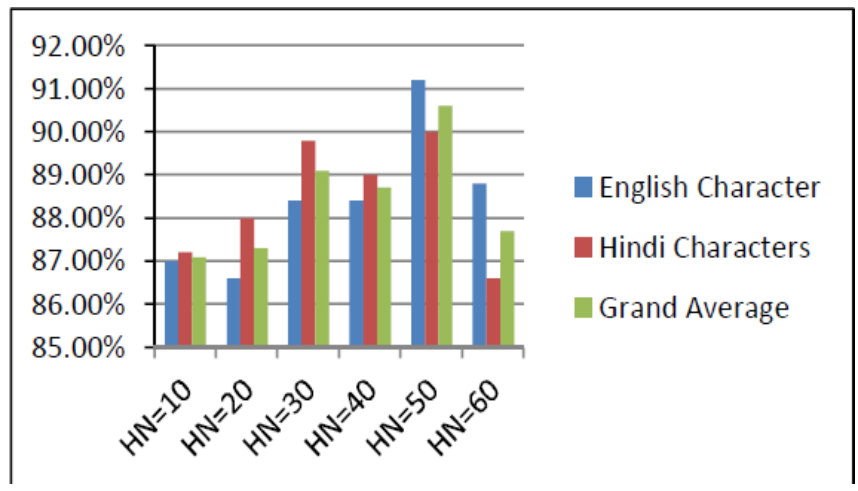

Fig. 5: Comparative network performances for image Dimension $=20 \times 20$ for Typewritten 79x10samples 


\section{CONCLUSIONS}

In this paper, I have presented a character recognition system and have shown the improvements achieved during this network implementation and its testing over real data. The important findings of the work may be summarized as follows:

In this work, I have implemented a character recognition system using feed forward back propagation network.

A sufficient collection of characters written in different font (hand written and type written) styles were kept for training this network and some type-written as well as handwritten data was kept for testing these networks.

This work has shown an improvement in performance of Feed Forward back-propagation Network and achieved more than $80 \%$ performance for handwritten samples for which system was untrained.

This work has still a good scope for future work. Some of the possibilities in this direction are as follows:

- The network has been worked only with 10 characters ( 5 characters of English and 5 character of Hindi) due to processing limit of system. The network can be further analyzed as well as enhanced to get similar performance for a larger range of characters.

- In today's environment, the possibilities are there for a mixed set of characters where more than one type of language can be used in day to day business. Keeping that in view, system can also be improved for Multilanguage environment. However, the main limitation is again of high speed of development platform.

- I have gone with improving of the single network i.e. the back propagation network. The comparison may be done with few other networks used in classification problem.

- The network can be further trained over a much larger data set.

\section{REFERENCES}

[1]. Pranob K Charles, V. Harish, M. Swathi and CH. Deepti, "A Review on the Various Techniques used for Optical Character Recognition", International Journal of Engineering Research and Applications(IJERA), ISSN: 2248-9622, Vol. 2 Issue 1, Jan-Feb 2012, pp.659-662.

[2]. Sumedha B. Hallale and Geeta D. Salunke, "Offline Handwriting Charater Recognition : A Review", International Journal of Advanced Computer and Mathematical Sciences, ISSN 2230-9624, Vol. 4, Issue 4, 2013, pp250-265.

[3]. Kauleshwar Prasad, Devrat C. Nigam, Ashmika Lakhotiya and Dheeren Umre, "Character Recognition Using Matlab's Neural Network Toolbox”, International Journal of u- and e- Service, Science and Technology, Vol. 6, No.1 , February, 2013.

[4]. Neha Sahu and Nitin Kali Raman, "An Efficient Handwritten Devnagari Character Recognition System
Using Neural Network", International Multi- Conference on Automation, Computing, Communication, Control and Compressed Sensing (iMac4s), 2013, Page(s):173-177.

[5]. Kunal Ravindra Shah and Dipak Dattatray Badgujar, "Devnagari Handwritten Character Recognition(DHCR) for Ancient Documents: A Review", Proceedings of 2013 IEEE Conference on Information and Communication Technologies (ICT 2013).

[6]. Kandarpa Kumar Sarma, "Bi-lingual Handwritten Character and Neural Recognition Using MultiDimensional Recurrent Neural Networks (MDRNN)", World Academy of Sciences, Engineering and Technology 282009.

[7]. Rajesh Soni and Durga Puja, "Performance Evaluation of Multilayer Feed Forward Neural for handwritten English Vowels Characters", International Conference on Information Systems and Computer Networks (ISCON), 2013, Page(s) : 82-87.

[8]. Dr. H. B. Kekre, Dr. Sudeep D. Thepade, Shrikant P.Sanas and Swapnil Shinde, "Devnagari Handwritten Character Recognition using LBG Vector Quantization with Gradient Masks", International Conference on Advances in Technology and Engineering (ICATE), 2013, Page(s):-1-4. [9]. Sandhya Arora, Debotosh Bhattacharjee, Mita Nasipuri, Dipak Kumar Basu and Mahantapas Kundu, "Multiple Classifier Combination for Offline Handwritten Devngari Character Recognition", International Confrence on Emerging Trends in Engineering and Technology (ICETET09) IEEE, DEC 2009. 